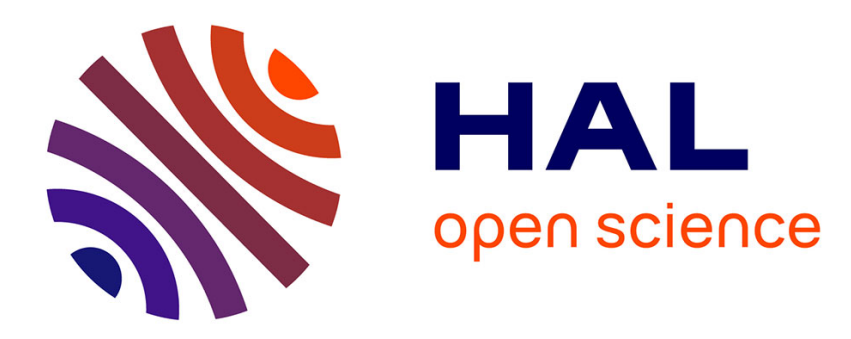

\title{
Modeling the impact of on-line navigation devices in traffic flows
}

\author{
Adriano Festa, Paola Goatin
}

\section{To cite this version:}

Adriano Festa, Paola Goatin. Modeling the impact of on-line navigation devices in traffic flows. CDC 2019 - 58th IEEE Conference on Decision and Control, Dec 2019, Nice, France. hal-02379576

\section{HAL Id: hal-02379576 \\ https://hal.science/hal-02379576}

Submitted on 25 Nov 2019

HAL is a multi-disciplinary open access archive for the deposit and dissemination of scientific research documents, whether they are published or not. The documents may come from teaching and research institutions in France or abroad, or from public or private research centers.
L'archive ouverte pluridisciplinaire HAL, est destinée au dépôt et à la diffusion de documents scientifiques de niveau recherche, publiés ou non, émanant des établissements d'enseignement et de recherche français ou étrangers, des laboratoires publics ou privés. 


\title{
Modeling the impact of on-line navigation devices in traffic flows
}

\author{
Adriano Festa and Paola Goatin
}

\begin{abstract}
We consider a macroscopic multi-population traffic flow model on networks accounting for the presence of drivers (or autonomous vehicles) using navigation devices to minimize their instantaneous travel cost to destination. The strategic choices of each population differ in the degree of information about the system: while part of the agents knows only the structure of the network and minimizes the traveled distance, others are informed of the current traffic distribution, and can minimize their travel time avoiding the most congested areas. In particular, the different route choices are computed solving eikonal equations on the road network and they are implemented at road junctions. The impact on traffic flow efficiency is illustrated by numerical experiments. We show that, even if the use of routing devices contributes to alleviate congestion on the whole network, it also results in increased traffic on secondary roads. Moreover, the generalized use of real-time information can even deteriorate the efficiency of the network.
\end{abstract}

\section{INTRODUCTION}

In recent years, navigation devices, such as Google Maps or Waze, have become very common in road users' daily life. This change modified consistently some aspects of traffic fluxes in many urban areas (cf. [15]). Besides the obvious benefits for the single user, recent studies [25], [17] have pointed out some drawbacks generated by selfish route choices in terms of traffic increase on the secondary network, in agreement with concerns expressed by local communities [11], [1]. This paper aims to provide the tools to address this problem in a macroscopic, non-stationary, simulation framework.

Macroscopic multi-population flow traffic models have been the subject of active research in recent years, mainly aiming at describing the interactions among different classes of vehicles such as cars and trucks (see, e.g. [2], [6]), cars and motorcycles [20], [9], [14] or even shared human and autonomous vehicles roads [18]. Some works also focus on the dynamics of several populations on a network, e.g. [26]. In particular, in [13] each population is identified by its origin and destination, while [7], [23] describe populations moving on predefined paths. On the contrary, here we are interested in modeling the case of drivers adapting their route dynamically to minimize their travel times based on the current state of the network.

\footnotetext{
*This work was partially supported by Societé Mathématiques Appliquée et Industriélles (SMAI) via the BOUM grant 2018.

A. Festa is with Dipartimento di Ingegneria, Scienze dell'Informazione e Matematica, Università dell'Aquila, Via Vetoio, 67100 L'Aquila, Italy, adriano.festalunivaq.it

P. Goatin is with Inria Sophia Antipolis - Méditerranée, Université Côte d'Azur, Inria, CNRS, LJAD, 2004, route des Lucioles - BP 9306902 Sophia Antipolis Cedex, FRANCE paola.goatindinria.fr
}

A similar problem has been addressed in the case of pedestrian flows: in [16], Hughes introduced a now classical model describing the motion of a large human crowd, seen as a "thinking fluid". The model expresses the fact that people seek to minimize their travel time to destination, trying to avoid high-density regions. This information is embedded in a potential field, which gives the direction of the motion. Indeed, the potential field is the solution of an eikonal equation, whose characteristic curves are the optimal paths to the destination, and whose running cost is proportional to the local crowd density. A discrete version of this model had been recently extended to networks [4].

In this paper, we propose a multi-population traffic flow model on networks, in which the flow of each population is governed by the corresponding mass conservation equation on road segments (summing up into the standard Lighthill-Williams-Richards (LWR) model [19], [21]). The conservation equations are coupled with as many eikonal equations on the underlying directed graph, whose running costs characterize each population and may depend on the traffic density. In this way, different levels of information can be taken into account, resulting in possibly time-dependent split ratios at road junctions. We note that, unlike the meanfield games framework, we do not consider any forecast ability for the future states of the system. The selected equilibrium can be seen, instead, as an adaptation of Hughes' pedestrian model to road networks.

The implementation of our model allows investigating several important points: Which is the evolution of the network global efficiency as the percentage of the routed vehicles increases? Is the presence of routed vehicles beneficial even for the less informed group? Which is the optimal percentage of vehicles provided of on-line navigation systems to minimize the overall congestion of the network? What is the impact on secondary roads? The numerical tests presented in Section $\mathrm{V}$ allow to qualitatively answer to the above questions.

\section{A GENERAL MULTI-CLASS TRAFFIC FLOW MODEL ON NETWORKS}

We introduce the general framework used for describing the flow of different classes of users on a road network. Each class density $\rho^{c}, c=1, \ldots, N_{c}$, is characterized by specific, possibly time-dependent, split ratios at junctions, depending on its degree of information about the network, see Section III.

Definition 2.1: An oriented network $\mathcal{N}=(\mathcal{I}, \mathcal{J})$ is a finite collection of points $\mathcal{J}:=\left\{J_{k}\right\}_{k \in \mathcal{K}}$ in $\mathbb{R}^{2}$ connected by continuous, non self-intersecting edges $\mathcal{I}:=\left\{I_{\ell}\right\}_{\ell \in \mathcal{L}}$, where $\mathcal{L}:=\{1, \ldots, N\}$ and $\mathcal{K}:=\{1, \ldots, M\}$. Each edge $I_{\ell} \in \mathcal{I}$ 
is parametrized by a smooth function $\pi_{\ell}:\left[0, L_{\ell}\right] \rightarrow \mathbb{R}^{2}$, $L_{\ell}>0$, which implicitly provides the orientation of the edge. Given $J_{k} \in \mathcal{J}, \operatorname{Inc}\left(J_{k}\right):=\left\{i \in \mathcal{L}: J_{k} \in I_{i}, \pi_{i}\left(L_{i}\right)=J_{k}\right\}$ denotes the set of edges arriving at $J_{k}$. Similarly, Out $\left(J_{k}\right):=$ $\left\{j \in \mathcal{L}: J_{k} \in I_{j}, \pi_{j}(0)=J_{k}\right\}$ denotes the set of edges leaving $J_{k}$. We define a subset $\mathcal{T} \subset \mathcal{J}$ of destination vertexes. We require that $\mathcal{T} \neq \emptyset$, i.e. the set $\mathcal{T}$ contains at least one element.

For any function $f: \mathcal{I} \rightarrow \mathbb{R}, f_{\ell}:\left[0, L_{\ell}\right] \rightarrow \mathbb{R}$ is the restriction of $f$ to $I_{\ell}$, i.e. $f(x)=f_{\ell}(y)$ for $x \in I_{\ell}$, $y=\pi_{\ell}^{-1}(x)$.

The dynamics of each class $\left.\rho^{c}:\right] 0, L_{\ell}[\times[0, T] \rightarrow[0,1]$ on each road $I_{\ell}$ is given by the mass conservation equation

$$
\left.\partial_{t} \rho_{\ell}^{c}+\partial_{x}\left(\rho_{\ell}^{c} v_{\ell}\left(\rho_{\ell}\right)\right)=0, \quad t>0, x \in\right] 0, L_{\ell}[,
$$

where $\rho_{\ell}(x, t):=\sum_{c=1}^{N_{c}} \rho_{\ell}^{c}(x, t)$ represents the total traffic density on the road $I_{\ell}$. Summing up the equations (1) for $c=1, \ldots, N_{c}$, we obtain the classical LWR equation

$$
\left.\partial_{t} \rho_{\ell}+\partial_{x}\left(\rho_{\ell} v_{\ell}\left(\rho_{\ell}\right)\right)=0, \quad t>0, x \in\right] 0, L_{\ell}[.
$$

In (1) and (2), the speed function $v_{\ell}:[0,1] \rightarrow\left[0, V_{\ell}\right]$ is a non-increasing function such that $v_{\ell}(0)=V_{\ell}$ and $v_{\ell}(1)=$ 0 (without loss of generality, we have normalized to 1 the maximal traffic density that can be attained on each road). Moreover, we assume that there exists a unique point $\hat{\rho}_{\ell} \in$ ] 0,1 [ such that the flux function $\rho \mapsto \rho v_{\ell}(\rho)$ is increasing for $\rho \in\left[0, \hat{\rho}_{\ell}[\right.$ and decreasing for $\left.\rho \in] \hat{\rho}_{\ell}, 1\right]$. We set $\gamma_{\ell}^{\max }:=$ $\hat{\rho}_{\ell} v_{\ell}\left(\hat{\rho}_{\ell}\right)=\max _{\rho \in I_{\ell}} \rho v_{\ell}(\rho)$ the maximal flux allowed on the $\operatorname{road} I_{\ell}$.

\section{A. Junction conditions for a single class model}

Let us now focus on the dynamics at junctions. We focus initially on a single population case. For any junction $J$, let us denote by $I_{i}, i=1, \ldots, n$, the incoming roads and by $I_{j}, j=n+1, \ldots, n+m$, the outgoing ones. The Riemann problem the junction $J$ denotes the Cauchy problem with constant initial data

$$
\left\{\begin{array}{l}
\partial_{t} \rho_{\ell}+\partial_{x}\left(\rho_{\ell} v_{\ell}\left(\rho_{\ell}\right)\right)=0, \\
\rho_{\ell}(\cdot, 0)=\rho_{0, \ell} \in[0,1],
\end{array} \quad \ell \in\{1, \ldots, n+m\} .\right.
$$

Given any distribution matrix $A=\left\{a_{j i}\right\} \quad i=1, \ldots, n$ such that $0 \leq a_{j i} \leq 1$, for any $i, j$ and $\sum_{j=n+1}^{n+m} a_{j i}=1$ for all $i$, we define a generic Riemann solver at $J$ as follows:

Definition 2.2: A Riemann solver $\mathcal{R S}_{A}$ is a function

$$
\begin{aligned}
\mathcal{R S}_{A}: \quad[0,1]^{n+m} & \longrightarrow \prod_{\ell=1}^{n+m}\left[0, \gamma_{\ell}^{\max }\right] \\
\left(\rho_{1}, \ldots, \rho_{n+m}\right) & \longmapsto\left(\bar{\gamma}_{1}, \ldots, \bar{\gamma}_{n+m}\right)
\end{aligned}
$$

where

$$
\left(\bar{\gamma}_{1}, \ldots, \bar{\gamma}_{n+m}\right):=\left(\bar{\rho}_{1} v_{1}\left(\bar{\rho}_{1}\right), \ldots, \bar{\rho}_{n+m} v_{n+m}\left(\bar{\rho}_{n+m}\right)\right)
$$

and such that

1) $\left(\bar{\gamma}_{n+1}, \ldots, \bar{\gamma}_{n+m}\right)^{T}=A \cdot\left(\bar{\gamma}_{1}, \ldots, \bar{\gamma}_{n}\right)^{T}$;

2) $\sum_{i=1}^{n} \bar{\gamma}_{i}=\sum_{j=n+1}^{n+m} \bar{\gamma}_{j}$;
3) for every $i=1, \ldots, n$, the classical Riemann problem

$$
\left\{\begin{array}{l}
\partial_{t} \rho+\partial_{x}\left(\rho v_{i}(\rho)\right)=0, \quad t>0, x \in \mathbb{R}, \\
\rho(\cdot, 0)= \begin{cases}\rho_{0, i} & x<0, \\
\bar{\rho}_{i} & x>0,\end{cases}
\end{array}\right.
$$

is solved with waves with negative speeds;

4) for every $j=n+1, \ldots, n+m$, the classical Riemann problem

$$
\left\{\begin{array}{l}
\partial_{t} \rho+\partial_{x}\left(\rho v_{j}(\rho)\right)=0, \quad t>0, x \in \mathbb{R}, \\
\rho(\cdot, 0)= \begin{cases}\bar{\rho}_{j} & x<0, \\
\rho_{0, j} & x>0,\end{cases}
\end{array}\right.
$$

is solved with waves with positive speeds.

Moreover, we require that $\mathcal{R S}_{A}$ satisfy the consistency condition

$$
\mathcal{R S}_{A}\left(\bar{\rho}_{1}, \ldots, \bar{\rho}_{n+m}\right)=\left(\bar{\gamma}_{1}, \ldots, \bar{\gamma}_{n+m}\right) .
$$

The Riemann solver provides the incoming/outgoing fluxes at the junction points satisfying the basic properties of conservation of mass and distribution of fluxes (see the recent monograph [12] for details). In the present work, we will use the Priority Riemann Solver introduced in [8], which can handle an arbitrary number of incoming and outgoing roads, accounting for priorities among the incoming roads and maximizing the through flux.

\section{B. Junction conditions for a multi-class model}

To extend the single class treatment of a junction to a multi-class framework, we generalize the multi-commodity case discussed in [22], [23] to any underlying Riemann solver $\mathcal{R S}_{A}$ satisfying Definition 2.2 This allows to consider junctions with any number of incoming and outgoing roads. The technique consists of the following steps:

1) Compose the total distribution matrix. Let the distribution matrices $A^{c}=\left\{a_{j i}^{c}\right\}_{i, j} \in \mathcal{A}$ at $\mathrm{J}$ be given for each class $\rho^{c}, c=1, \ldots, N_{c}$ (these coefficients will be derived in Section [III). We set

$$
A:=\left\{a_{j i}\right\}, \quad \text { where } \quad a_{j i}:=\sum_{c=1}^{N_{c}} a_{j i}^{c} \frac{\rho_{i}^{c}}{\rho_{i}}
$$

defines a weighted distribution matrix for the total density of the populations at the junction.

2) Compute the fluxes. Using the selected Riemann solver $\mathcal{R S}_{A}$ corresponding to (4), we obtain the total outgoing fluxes $\left(\bar{\gamma}_{1}, \ldots, \bar{\gamma}_{n+m}\right)$.

3) Distribute the fluxes among the various classes. The incoming and outgoing fluxes for each class are given by

$$
\begin{gathered}
\bar{\gamma}_{i}^{c}=\frac{\rho_{i}^{c}}{\rho_{i}} \bar{\gamma}_{i}, \quad i=1, \ldots, n, \quad c=1, \ldots, N_{c}, \\
\bar{\gamma}_{j}^{c}=\sum_{i=1}^{n} a_{j i}^{c} \bar{\gamma}_{i}^{c}, \quad j=n+1, \ldots, n+m .
\end{gathered}
$$




\section{STRATEGY MODELING ON THE NETWORK}

In order to model the strategic route choices of the vehicles, we use dynamic programming, which characterises the value function of an optimal control problem defined on the network as a solution of a Hamilton-Jacobi (HJ) equation. Viscosity solutions for $\mathrm{HJ}$ equations on networks have been introduced in recent years (see e.g. [12], [24], [3]), the case of the directed graph - where the arcs can be traveled only in one direction - can be considered a sub-case of the latter.

We start defining formally the optimal control problem on a graph. We denote by $\Upsilon_{z, x}$ the set of absolute continuous curves connecting $z$ to $x \in \mathcal{T}^{c}$, identified by a finite sequence $\ell_{n} \in \mathcal{L}, n=0, \ldots, N_{\Upsilon}$, such that there exists a $\hat{z} \in\left[0, L_{\ell_{0}}\right]$ with $\pi_{\ell_{0}}(\hat{z})=z, \pi_{\ell_{i}}(0)=\pi_{\ell_{i-1}}\left(L_{\ell_{i-1}}\right), i=1, \ldots, N_{\Upsilon}$, $\pi_{\ell_{N_{\Upsilon}}}\left(L_{\ell_{N_{\Upsilon}}}\right)=x$. Then the value function of the optimal control problem that minimizes the weighted distance from a target set $\mathcal{T}^{c}$, minimizing the running cost $g^{c}:[0,1] \rightarrow \mathbb{R}^{+}$ (which we assume to be a Lipschitz continuous function), is given by

$$
u_{\ell}^{c}(y)=\inf \left\{d_{c}\left(x, \pi_{\ell}(y)\right): x \in \mathcal{T}^{c}\right\},
$$

where the distance function $d_{c}: \mathcal{I} \times \mathcal{I} \rightarrow \mathbb{R}^{+}$is

$$
\begin{array}{r}
d_{c}(x, z)=\inf _{\left(\ell_{0}, \ldots, \ell_{N_{\Upsilon}}\right) \in \Upsilon_{z, x}}\left\{\int_{\hat{z}}^{L_{\ell}} \frac{1}{g^{c}\left(\rho_{\ell}\left(\pi_{\ell}(s), t\right)\right)} \mathrm{d} s\right. \\
\left.+\sum_{i=1}^{N_{\Upsilon}} \int_{0}^{L_{\ell_{i}}} \frac{1}{g^{c}\left(\rho_{\ell_{i}}\left(\pi_{\ell_{i}}(s), t\right)\right)} \mathrm{d} s\right\} .
\end{array}
$$

We remark that the function $g^{c}$ models the influence of the traffic density in the choice of the "shortest" path for the $c$-th vehicle class. In general, the function (5) can be not defined on some arcs, since a minimal path (i.e., an itinerary to reach a destination vertex in an optimal way) could be not defined in some areas of the network. To overcome this problem, we make the following hypothesis:

For every $z \in \mathcal{I}$ there exists at least one $x \in \mathcal{T}^{c}$ such that the set $\Upsilon_{z, x} \neq \emptyset$, i.e. there exists a sequence of indexes $\ell_{0}, \ldots, \ell_{N_{\Upsilon}}$ such that $z \in I_{\ell_{0}}, \pi_{\ell_{i-1}}\left(L_{\ell_{i-1}}\right)=\pi_{\ell_{i}}(0)$ for $i=1, \ldots, N_{\Upsilon}$ and $\pi_{\ell_{N_{\Upsilon}}}\left(L_{\ell_{N_{\Upsilon}}}\right)=x \in \mathcal{T}^{c}$.

Using the dynamic programming principle, we observe that the value function $u_{\ell}^{c}$ defined in (5) is the viscosity solution of

$$
\begin{cases}\partial_{x} u_{\ell}^{c}(x)+\frac{1}{g^{c}\left(\rho_{\ell}(x, t)\right)}=0 & x \in] 0, L_{\ell}[, \ell \in \mathcal{L}, \\ \min _{\ell \in O u t\left(J_{k}\right)} u_{\ell}^{c}(0)=u_{l}^{c}\left(L_{l}\right) & J_{k} \in \mathcal{J} \backslash \mathcal{T}, l \in \operatorname{Inc}\left(J_{k}\right) \\ u_{\ell}^{c}\left(L_{\ell}\right)=0, & \pi_{\ell}\left(L_{\ell}\right) \in \mathcal{T}^{c},\end{cases}
$$

which gives the optimal path leading to the target set $\mathcal{T}^{c}$, depending on the perceived cost $g^{c}$. Since the arcs are oriented, route choices are possible only at junctions. In particular, this allows to define the matrix distribution coefficients in (4). For each junction $J_{k}$, we define the set

$$
W_{k}^{c}:=\left\{l \in \operatorname{Out}\left(J_{k}\right): \quad u_{l}^{c}(0)=\min _{j \in O u t\left(J_{k}\right)} u_{j}^{c}(0)\right\},
$$

denoting by $\left|W_{k}^{c}\right|$ its cardinality. We define the distribution matrix $A_{k}^{c}=\left\{\alpha_{j i}^{c}\right\}_{j i}$ of the population $c$ at $J_{k}$ as

$$
\alpha_{j i}^{c}= \begin{cases}1 /\left|W_{k}^{c}\right|, & \text { if } j \in W_{k}^{c} \\ 0, & \text { otherwise. }\end{cases}
$$

In other words, we dynamically adapt the distribution matrix to the optimal options at any junction. In particular, we assign equal weight to all the equally profitable options.

The destination vertexes act as additional conditions imposed at the boundary (cf. [10]), motivating the choice of setting the potential $u^{c}$ to zero at these points.

Remark 3.1 (2-population case): We describe in detail the special case of two populations of vehicles $\rho^{1}$ and $\rho^{2}$ with the same destination set $\left(\mathcal{T}^{1}=\mathcal{T}^{2}\right)$, as considered in the simulation Section $\mathrm{V}$. While we fix, for any choice of $\ell \in \mathcal{L}, v_{\ell}^{1}(\rho)=v_{\ell}^{2}(\rho)=1-\rho$, we choose $g^{1}(\rho)=1$ and $g^{2}(\rho)=1-\rho$. In this way, the two populations' route choices - despite the two groups have the same destination - may be different at the junctions. In particular, the above choices of $g^{1}$ and $g^{2}$ model the case where the first population does not use any on-line routing device. The knowledge of the network - or the use of maps or off-line routing devices - leads the density $\rho^{1}$ to follow the shortest path to the destination. On the contrary, $\rho^{2}$ uses information on the current state of the system to avoid congested regions, thus minimizing the time to destination.

\section{A DISCRETE MULTI-CLASS, MULTI-INFORMED TRAFFIC FLOW MODEL ON ORIENTED NETWORKS}

Let us consider a standard discretization of the network $\mathcal{N}$, where a generic edge $I_{\ell}$ is approximated by $N_{\ell}$ points. Setting $\delta_{\ell}=L_{\ell} /\left(N_{\ell}-1\right)$, we define the space grid points $x_{\ell, h}=\pi_{j}\left((h-1) \delta_{\ell}\right)$ for $h=1, \ldots, N_{\ell}$, and $\Delta x_{\ell, h}:=\left|x_{\ell, h+1}-x_{\ell, h}\right|$. For each junction point $J_{k} \in \mathcal{J}$, we denote by $\operatorname{Inc}\left(J_{k}\right)=\left\{\ell_{1}, \ldots, \ell_{n_{k}}\right\}$ and $\operatorname{Out}\left(J_{k}\right)=$ $\left\{\ell_{n_{k}+1}, \ldots, \ell_{n_{k}+m_{k}}\right\}$ the indexes of incoming and outgoing roads, respectively.

For any given initial distribution $\bar{\rho}^{c}: \mathcal{I} \rightarrow[0,1]$, the multi-class traffic flow dynamics on the network $\mathcal{N}$ is then described by following discrete system for a $\nu \in \mathbb{N}, \ell \in \mathcal{L}$, $h=2, \ldots, N_{\ell}-1$ and $c=1, \ldots, N_{c}$ :

$$
\begin{aligned}
& \rho_{\ell, 1}^{c, \nu+1}=\rho_{\ell, 1}^{c, \nu}-\frac{\Delta t}{\Delta x_{\ell, 1}}\left(\frac{\rho_{\ell, 1}^{c, \nu}}{\rho_{\ell, 1}^{\nu}} F_{\ell, 1}^{\nu}-\bar{\gamma}_{\ell, 1}^{c, \nu}\right), \\
& \rho_{\ell, h}^{c, \nu+1}=\rho_{\ell, h}^{c, \nu}-\frac{\Delta t}{\Delta x_{\ell, h}}\left(\frac{\rho_{\ell, h}^{c, \nu}}{\rho_{\ell, h}^{\nu}} F_{\ell, h}^{\nu}-\frac{\rho_{\ell, h-1}^{c, \nu}}{\rho_{\ell, h-1}^{\nu}} F_{\ell, h-1}^{\nu}\right), \\
& \rho_{\ell, N_{\ell}}^{c, \nu+1}=\rho_{\ell, N_{\ell}}^{c, \nu}-\frac{\Delta t}{\Delta x_{\ell, N_{\ell}}}\left(\bar{\gamma}_{\ell, N_{\ell}}^{c, \nu}-\frac{\rho_{\ell, N_{\ell}-1}^{c, \nu}}{\rho_{\ell, N_{\ell}-1}^{\nu}} F_{\ell, N_{\ell}-1}^{\nu}\right), \\
& \frac{u_{\ell, h+1}^{c, \nu}-u_{\ell, h}^{c, \nu}}{\Delta x_{\ell, h}}+\frac{1}{g^{c}\left(\rho_{\ell, h}^{\nu}\right)}=0, \\
& u_{\ell, N_{\ell}}^{c, \nu}=\min _{i \in O u t\left(J_{k}\right)} u_{i, 1}^{c, \nu}, x_{\ell, N_{\ell}}=J_{k} \in \mathcal{J},
\end{aligned}
$$




$$
\begin{aligned}
& W_{k}^{c, \nu}=\left\{l \in \operatorname{Out}\left(J_{k}\right): u_{l, 1}^{c, \nu}=\min _{i \in \operatorname{Out}\left(J_{k}\right)} u_{i, 1}^{c, \nu}\right\}, \\
& A_{k}^{c, \nu}=\left\{a_{j i}^{c, \nu}\right\}_{j i}: a_{j i}^{c, \nu}= \begin{cases}1 /\left|W_{k}^{c, \nu}\right|, & \text { if } j \in W_{k}^{c, \nu}, \\
0, & \text { otherwise, }\end{cases} \\
& A_{k}^{\nu}=\left\{\sum_{c=1}^{N_{c}} a_{j i}^{c, \nu} \frac{\rho_{i, N_{i}}^{c, \nu}}{\rho_{i, N_{i}}^{\nu}}\right\}_{j i}, \\
& \left(\bar{\gamma}_{\ell_{1}}^{\nu}, \ldots, \bar{\gamma}_{\ell_{n_{k}+m_{k}}}^{\nu}\right)=\mathcal{R S}_{A_{k}^{\nu}}\left(\rho_{\ell_{1}}^{\nu}, \ldots, \rho_{\ell_{n_{k}+m_{k}}}^{\nu}\right) \\
& \bar{\gamma}_{i, N_{i}}^{c, \nu}=\frac{\rho_{i, N_{i}}^{c, \nu}}{\rho_{i, N_{i}}^{\nu}} \bar{\gamma}_{i}^{\nu}, \quad i \in \operatorname{Inc}\left(J_{k}\right), \\
& \bar{\gamma}_{j, 1}^{c, \nu}=\sum_{i=\ell_{1}}^{\ell_{n_{k}}} a_{j i}^{c, \nu} \bar{\gamma}_{i}^{c, \nu}, \quad j \in O u t\left(J_{k}\right), \\
& \rho_{\ell, h}^{c, 0}=\frac{1}{\Delta x_{\ell, h}} \int_{x_{\ell, h}}^{x_{\ell, h+1}} \bar{\rho}_{\ell}^{c}(x) d x, \\
& u_{\ell, N_{\ell}}^{c, \nu}=\rho_{\ell, N_{\ell}}^{c, \nu}=0, \quad x_{\ell, N_{\ell}} \in \mathcal{T}^{c},
\end{aligned}
$$

In (9), the numerical flux $F_{\ell, h}^{\nu}$ is the standard Godunov flux corresponding to 22, defined by

$$
F_{\ell, h}^{\nu}=F_{\ell}\left(\rho_{\ell, h}^{\nu}, \rho_{\ell, h+1}^{\nu}\right):=\min \left\{D_{\ell}\left(\rho_{\ell, h}^{\nu}\right), S_{\ell}\left(\rho_{\ell, h+1}^{\nu}\right)\right\},
$$

where $D_{\ell}(\rho)$ and $S_{\ell}(\rho)$ are the demand and supply functions defined by

$$
\begin{aligned}
& D_{\ell}(\rho)= \begin{cases}\rho v_{\ell}(\rho) & \text { if } \rho \in\left[0, \hat{\rho}_{\ell}\right], \\
\gamma_{\ell}^{\max } & \text { if } \rho \in\left[\hat{\rho}_{\ell}, 1\right],\end{cases} \\
& S_{\ell}(\rho)= \begin{cases}\gamma_{\ell}^{\max } & \text { if } \rho \in\left[0, \hat{\rho}_{\ell}\right], \\
\rho v_{\ell}(\rho) & \text { if } \rho \in\left[\hat{\rho}_{\ell}, 1\right],\end{cases}
\end{aligned}
$$

see for example [12], Section 5.2.3.

To guarantee the stability of the scheme 9), we impose that

$$
\Delta t \leq \min _{\ell, h} \frac{\Delta x_{\ell, h}}{V_{\ell}} .
$$

We also assume that the vehicles exit the network once they have reached their destination. In this way, they do not contribute further to the possible congestion effects. Alternative choices are represented by Neumann condition bounding the flux to a specific exit rate, or directly imposing some non-flux condition (see [4]).

\section{NUMERICAL RESULTS}

In the following tests, we consider two populations of vehicles. For the first one, we set $v_{\ell}^{1}(\rho)=(1-\rho), \ell \in \mathcal{L}$, and $g^{1}(\rho)=1$. Therefore, the agents belonging to the first population have a perfect knowledge of the geometry of the network, but they do not know the current traffic conditions. For the second population we set $v_{\ell}^{2}(\rho)=g^{2}(\rho)=(1-\rho)$. In this case, the drivers have real-time knowledge of the traffic condition of the network (as well as its geometrical features). They then choose an itinerary that minimizes the current time to reach the destination.
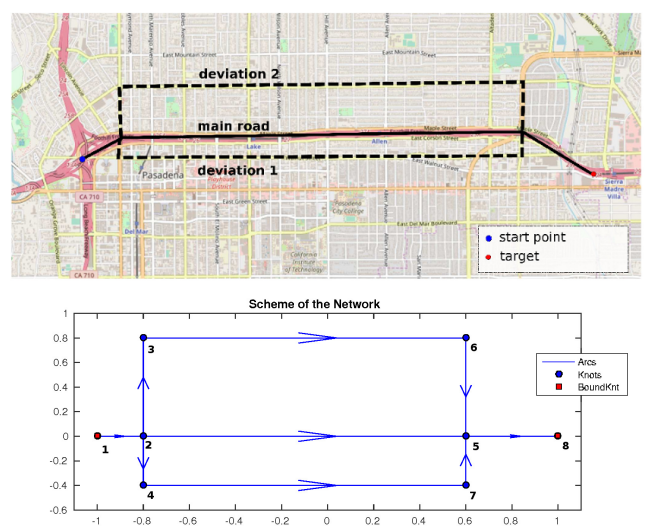

Fig. 1. The portion of Los Angeles road network considered in Section VA (top, credit "(C) OpenStreetMap contributors" Www . openstreetmap. org) and its schematic representation used for the numerical simulations.
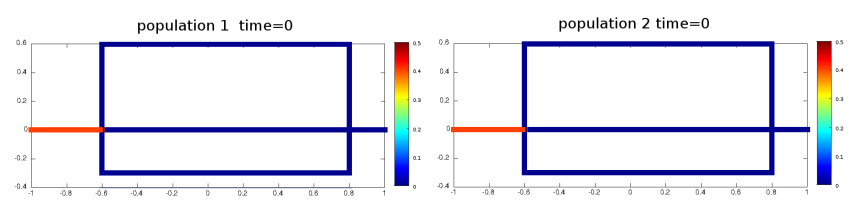

population 1 time $=1.2$

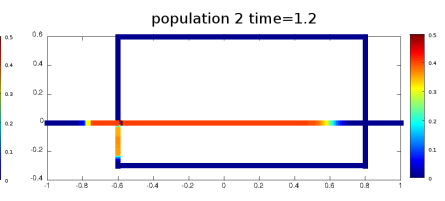

population 1 time $=2$
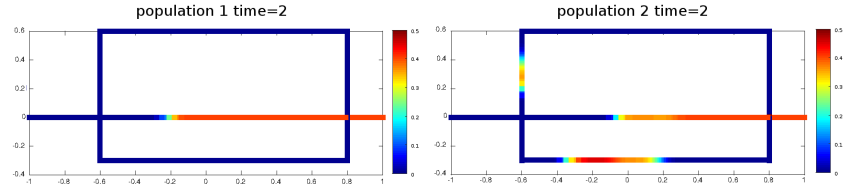

Fig. 2. Evolution of the densities $\rho^{1}$ corresponding to the non informed population (left) and $\rho^{2}$ of the informed one (right) for $\mathcal{P}=0.5$.

\section{A. A simple road network}

For a first qualitative analysis, we consider an elementary network composed of 8 junctions, which was studied in a static framework in [17]. It refers to a small portion of Los Angeles road network, where two secondary itineraries offer an alternative to the main road when it gets congested (see Fig. 11.

We fix the boundary conditions $\rho(x, \cdot)=0$ at the exit node n. 8, and zero incoming flux at node $\mathrm{n}$. 1 . We construct a uniform grid on the arcs with step $\Delta x=0.05$ and we sample the time with steps of size $\Delta t=0.01$. The initial condition $\bar{\rho}$ is set equal to zero in all the network except in the arc $1-2$, where it is set equal to 0.9 . The proportion between the two populations is given by a parameter $\mathcal{P} \in[0,1]$ as we set

$$
\bar{\rho}^{1}=(1-\mathcal{P}) \bar{\rho}, \quad \bar{\rho}^{2}=\mathcal{P} \bar{\rho} .
$$

At junction n. 5, we set equal priority parameters $p=$ $(1 / 3,1 / 3,1 / 3)$, meaning that vehicles coming from the main road and the secondary roads access arc 5-8 with the same priority. 

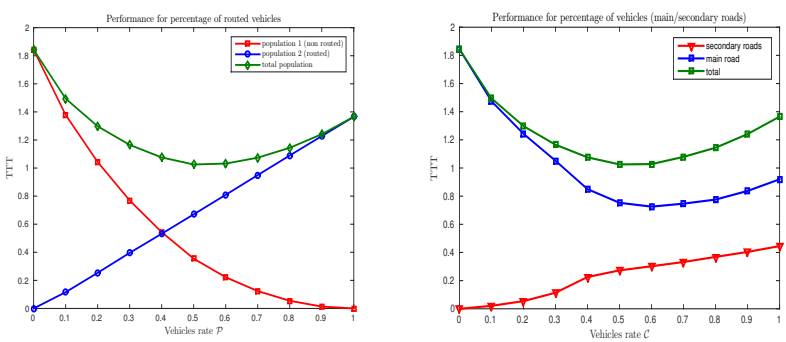

Fig. 3. (Left) TTT on the whole network for each of the two populations and for the total population, in the case of the network depicted in Figures 1 and 2 depending on the penetration rate of routed vehicles $\mathcal{P}$. (Right) TTT in the main road and in the two detours to reach destination as a function of the penetration rate of routed vehicles $\mathcal{P}$.

Figure 2 shows the traffic density evolution for the two populations. We remark that, for the first one, all the drivers opt for the shortest path (the itinerary 1-2-5-8) (Figure 2]left). The evolution of the second population is more complex. At the beginning, the shortest path $1-2-5-8$ is the most favorable option, but it becomes soon too crowded, and part of the population selects the route 1-2-4-7-5-8. When even this itinerary gets enough crowded to loose its benefits, part of the agents choose the last option 1-2-3-6-5-8, since the congestion level in the other routes justifies such detour (Figure 2 left).

Figure 3 (left) shows the dependence of the Total Travel Time (TTT) from the percentage of routed vehicles $\mathcal{P}$, where

$$
\operatorname{TTT}(\rho)=\Delta t \Delta x \sum_{\nu=0}^{N_{f}} \sum_{\ell \in \mathcal{L}} \sum_{h=1}^{N_{\ell}} \rho_{\ell, h}^{\nu}
$$

(i.e. the space/time integral of the density) relative to the two populations $\left(T T T\left(\rho^{1}\right)\right.$ and $\left.T T T\left(\rho^{2}\right)\right)$ and the total traffic $\left(T T T(\rho)=T T T\left(\rho^{1}+\rho^{2}\right)=T T T\left(\rho^{1}\right)+T T T\left(\rho^{2}\right)\right)$. In 14, we have set $N_{f}=T_{f} / \Delta t$, where $T_{f}=5$ is the simulation time.

It is clear that despite the TTT is computed in the whole time interval $\left[0, T_{f}\right]$, once all the vehicles have left the network, the value of the TTT stays constant. We also notice that, with the increase of the total number of routed vehicles, the global total travel time first decreases, reaching a minimum value around $\mathcal{P}=0.5$, but then increases again, probably due to the presence of congestions in the arc 5-8. This means that the presence of routed vehicles can indeed alleviate traffic congestion if their percentage is not too high, but in the limit scenario in which all cars use routing devices, the network efficiency could not be optimized in a global sense. For example, no vehicle would never accept a less convenient strategy to avoid the appearance of congestion in some section of the network. This is consistent with literature [17], indicating that a selfish behavior of vehicles brings to a Wardrop equilibrium [27], i.e. a configuration where no individual driver can reduce his path cost by a change of route. This kind of equilibrium is not in general globally optimal, but, since no unilateral change of strategy in any group of vehicles can lead to a reduction of the cost, it can be seen as Nash equilibrium among the agents [5].

In Figure 3 (right) we investigate another drawback generated by the use of routing devices, that is the increase of traffic on secondary roads, usually serving residential areas. This is in agreement with the study reported in [25] in a static framework.

\section{B. Sophia Antipolis road network}

We consider a more complex scenario derived from the road network in Sophia Antipolis (France), see Figure 4. Here the boundary nodes of the network are displayed in red. The nodes n. 1 and 5 correspond to the entrance/exit of the high capacity highway A8 La Provencale, while the node n. 30 is a parking area considered as the target in our simulation. The discretization step is set $\Delta x=5$ and the time step $\Delta t=0.8 \Delta x$.

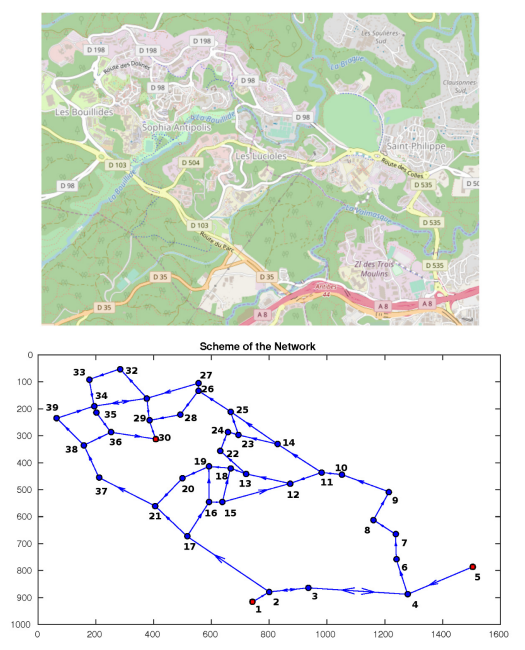

Fig. 4. Road network of the Sophia Antipolis area and Map (credit "(C) OpenStreetMap contributors" Www . openst reetmap.org).

In Figure 5 we can see the initial distribution of the density (we start with a constant distribution of vehicles $\bar{\rho}=0.8$ on the high capacity highway composed by the path 1-2-3-4-5) and its time evolution, for the two populations. We establish the percentage of the vehicles belonging to each class using, as in the previous case, the parameter $\mathcal{P} \in[0,1]$. As in the previous case, the priority parameters at junctions are chosen to be equal between the various incoming roads.

We observe the different strategies of the two populations: While population 1 points directly to the objective (node n. 30), reaching high-density levels on the shortest path to the destination, the second population splits since the beginning of the simulation into two main routes to reach the destination. It is interesting to notice that also several minor connections are used to balance the flux between the various paths chosen. As expected, the routed population tends to avoid the shorter but high congested path traveled by population 1 . 

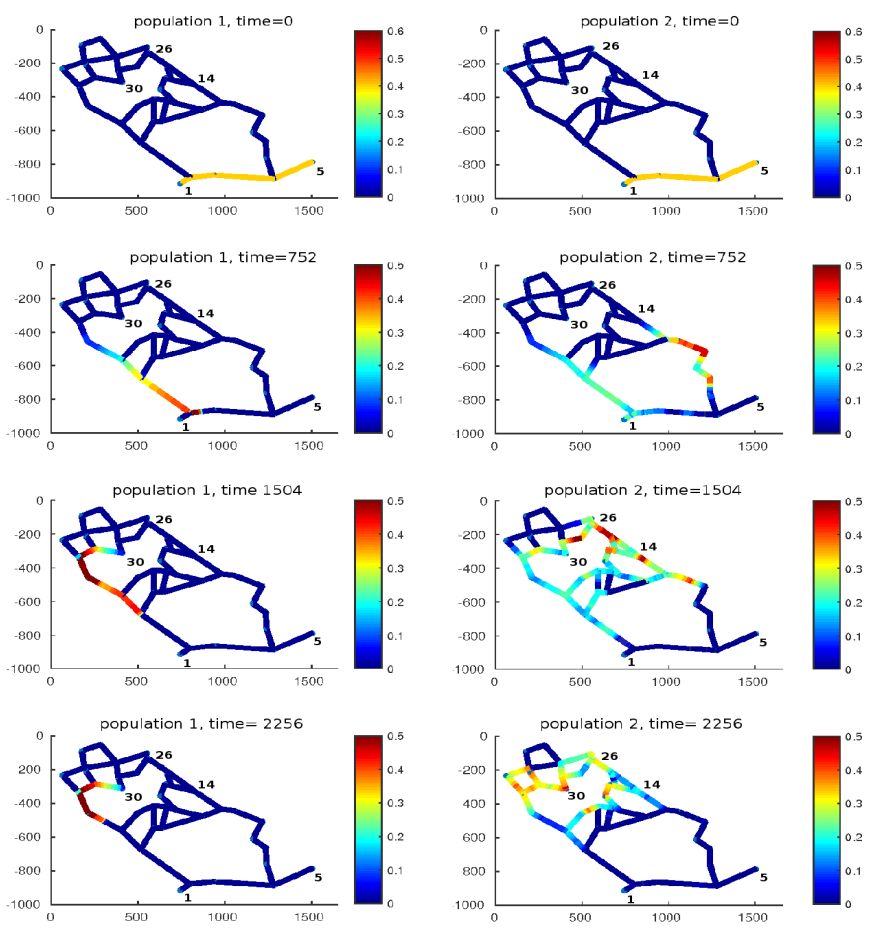

Fig. 5. Evolution of the density $\rho$ in the case of non informed population (left) and informed (right) for $\mathcal{P}=0.5$. The samples are taken at time $t=0,752,1504,2256$.

\section{CONCLUSIONS}

In this paper we have presented a new dynamic macroscopic multi-population traffic model on road networks, where the different population classes are characterised by their optimal strategies to reach a target. The strategies are different since they are deduced in base of information about the state of the system of every class. In particular, we have studied the impact on the whole traffic flow of different penetration rates of vehicles routed by on-line navigation devices. Our numerical experiments show that the total travel time of all users reaches a minimum, but then it increases again for large percentages of routed vehicles, driving the system to some form of Wardrop equilibrium point. Moreover, the traffic flow on secondary roads also increases as the number of routed vehicles increases.

This work intends to be a preliminary introduction of the model, since several aspects still need to be addressed. First of all, we plan to test the proposed approach in more realistic scenarios, possibly validating the results against real data. It would be also interesting to evaluate the impact of traffic navigation systems by comparing the network performances obtained using system optimal traffic assignment policies, see e.g. [23]. Finally, a substantial theoretical work of validation of the model is necessary, in particular regarding the nature of the selected equilibria between the agents.

\section{REFERENCES}

[1] J.-G. B. Circulation : Waze, l'appli qui agace les riverains. Le Parisien, Jun 2018.
[2] S. Benzoni-Gavage and R. M Colombo. An $n$-populations model for traffic flow. Eur. J. Appl. Math., 14(05):587-612, 2003.

[3] F. Camilli, A. Festa, and D. Schieborn. An approximation scheme for a Hamilton-Jacobi equation defined on a network. Appl. Num. Math., 73:33-47, 2013.

[4] F. Camilli, A. Festa, and S. Tozza. A discrete Hughes' model for pedestrian flow on graphs. Netw. Heterog. Media, 12(1): 93-112, 2017.

[5] G. Carlier and F. Santambrogio. A continuous theory of traffic congestion and Wardrop equilibria. J. Math. Sc., 181(6):792-804, 2012

[6] S. Chanut and C. Buisson. Macroscopic model and its numerical solution for two-flow mixed traffic with different speeds and lengths. Trans. Res. Rec., (1852):209-219, 2003.

[7] E. Cristiani and F. S. Priuli. A destination-preserving model for simulating Wardrop equilibria in traffic flow on networks. Netw. Heterog. Media, 10(4):857-876, 2015.

[8] M.-L. Delle Monache, P. Goatin, and B. Piccoli. Priority-based Riemann solver for traffic flow on networks. Commun. Math. Sci. 16(1):185-211, 2018

[9] S. Fan and D. B. Work. A heterogeneous multiclass traffic flow model with creeping. SIAM J. Appl. Math., 75(2):813-835, 2015.

[10] A. Festa, R. Guglielmi, C. Hermosilla, A. Picarelli, S. Sahu, A. Sassi, and F. J. Silva. Hamilton-Jacobi-Bellman Equations, pages 127-261. Springer International Publishing, Cham, 2017.

[11] L. W. Foderaro. Navigation apps are turning quiet neighborhoods into traffic nightmares. The New York Times, Dec 2017.

[12] M. Garavello, K. Han, and B. Piccoli. Models for vehicular traffic on networks, volume 9. American Institute of Mathematical Sciences (AIMS), Springfield, MO, 2016.

[13] M. Garavello and B. Piccoli. Source-destination flow on a road network. Commun. Math. Sci., 3(3):261-283, 2005.

[14] S. Gashaw, P. Goatin, and J. Härri. Modeling and analysis of mixed flow of cars and powered two wheelers. Transport. Res. Part C-Emerg. Technol., 89:148-167, 2018.

[15] H. Gharavi, K. V. Prasad, and P. Ioannou. Scanning advanced automobile technology. Proc. IEEE Inst. Electr. Electron. Eng., 95(2):328-333, 2007

[16] R. L. Hughes. A continuum theory for the flow of pedestrians Transport. Res. Part B-Meth., 36(6):507-535, 2002.

[17] A. Keimer, N. Laurent-Brouty, F. Farokhi, H. Signargout, V. Cvetkovic, A. M. Bayen, and K. H. Johansson. Information patterns in the modeling and design of mobility management services. Proc. IEEE Inst. Electr. Electron. Eng., 106(4):554-576, April 2018.

[18] M. W. Levin and S. D. Boyles. A multiclass cell transmission model for shared human and autonomous vehicle roads. Transport. Res. Part C-Emerg. Technol., 62:103-116, 2016.

[19] M. J. Lighthill and G. B. Whitham. On kinematic waves II. a theory of traffic flow on long crowded roads. Proc. R. Soc. Lond. A, 229(1178):317-345, 1955

[20] R. Nair, H. S. Mahmassani, and E. Miller-Hooks. A porous flow approach to modeling heterogeneous traffic in disordered systems. Transp. Res. B: Meth., 45(9):1331-1345, 2011.

[21] P. I. Richards. Shock waves on the highway. Operations Res., 4:42-51, 1956.

[22] S. Samaranayake, J. Reilly, W. Krichene, J. B. Lespiau, M. L. Delle Monache, P. Goatin, and A. Bayen. Discrete-time system optimal dynamic traffic assignment (SO-DTA) with partial control for horizontal queuing networks. In 2015 American Control Conference (ACC), pages 663-670, July 2015.

[23] S. Samaranayake, J. Reilly, W. Krichene, M.-L. Delle Monache, P. Goatin, and A. Bayen. Discrete-time system optimal dynamic traffic assignment (SO-DTA) with partial control for horizontal queuing networks. Transport. Sci., 2018

[24] D. Schieborn and F. Camilli. Viscosity solutions of eikonal equations on topological networks. Calc. Var. Partial Dif., 46(3-4):671-686, 2013

[25] J. Thai, N. Laurent-Brouty, and A. M. Bayen. Negative externalities of GPS-enabled routing applications: A game theoretical approach. In 2016 IEEE 19th International Conference on Intelligent Transportation Systems (ITSC), pages 595-601, Nov 2016.

[26] J. Van Lint, S. Hoogendoorn, and M. Schreuder. Fastlane: New multiclass first-order traffic flow model. Trans. Res. Rec., (2088):177187, 2008.

[27] J. G. Wardrop. Some theoretical aspects of road traffic research. In Inst. Civil Engineers Proc. London, 1952. 